\title{
On $m$-closed graphs
}

\author{
Leila Sharifan \\ Department of Mathematics \\ Hakim Sabzevari University \\ Sabzevar, Iran, P.O. Box 397 \\ leila-sharifan@aut.ac.ir
}

\author{
Masoumeh Javanbakht \\ Department of Mathematics \\ Hakim Sabzevari University \\ Sabzevar, Iran, P.O. Box 397 \\ masumehjavanbakht@gmail.com
}

Submitted: May 27, 2014; Accepted: Oct 24, 2014; Published: Nov 6, 2014

Mathematics Subject Classifications: 05C78, 05C25, 13P10, 05E40.

\begin{abstract}
A graph is closed when its vertices have a labeling by $[n]$ such that the binomial edge ideal $J_{G}$ has a quadratic Gröbner basis with respect to the lexicographic order induced by $x_{1}>\cdots>x_{n}>y_{1}>\cdots>y_{n}$. In this paper, we generalize this notion and study the so called $m$-closed graphs. We find equivalent condition to 3 -closed property of an arbitrary tree $T$. Using it, we classify a class of 3-closed trees. The primary decomposition of this class of graphs is also studied.
\end{abstract}

Keywords: $m$-closed graph, binomial edge ideal, reduced Gröbner basis, admissible path

\section{Introduction and Preliminaries}

Suppose $G$ is a simple graph on the vertex set $[n]$ and $R=\mathbf{k}\left[x_{1}, \ldots, x_{n}, y_{1}, \ldots, y_{n}\right]$ is the polynomial ring over the field $\mathbf{k}$. The binomial edge ideal of $G$ is the ideal

$$
J_{G}=\left(f_{i j}:\{i, j\} \in E(G) \text { and } i<j\right) \subset R,
$$

where $f_{i j}=x_{i} y_{j}-x_{j} y_{i}$. This notion was first introduced in [9] and independently in [13].

Note that any ideal generated by a set of 2 -minors of a $2 \times n$-matrix $X$ of indeterminates may be viewed as the binomial edge ideal of a graph. In [9], the authors compute the reduced Gröbner basis of the binomial edge ideal with respect to the lexicographic order induced by $x_{1}>\cdots>x_{n}>y_{1}>\cdots>y_{n}$ (we show this order by $\prec$ ). In particular, they find the necessary and sufficient conditions in which $J_{G}$ has a quadratic Gröbner basis. Graphs whose binomial edge ideal has a quadratic Gröbner basis are called closed graphs and the Cohen-Macaulay property of these graphs is studied in [6]. Recently, many authors studied the algebraic properties of some classes of binomial edge ideals. In 
particular the regularity and the depth are studied in $[1,6,7,10,12,15]$. But the reduced Gröbner basis obtained in [9] has not been studied in more details.

In this paper, we study the Gröbner basis of $J_{G}$ where $G$ is a simple graph. We call $G$ an $m$-closed graph when its vertices can be labeled by $[n]$ such that the elements of the reduced Gröbner basis of $J_{G}$ have degree at most $m$, and $m$ is the least integer with this property for $G$. Note that by this definition, a closed graph is a 2-closed graph.

In Section 2 we study some basic properties of $m$-closed graphs. In particular, we show

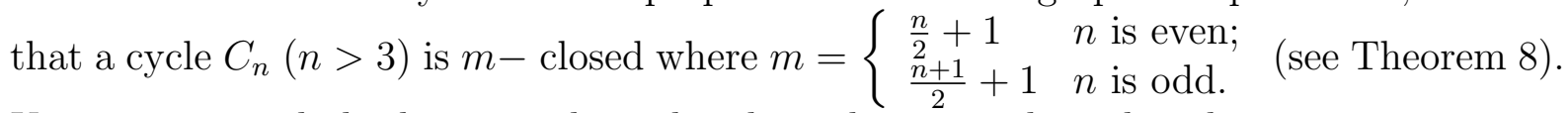
Using it we conclude that in each $m$-closed graph, any cycle with at least $2 m-1$ vertices has a chord.

The notion of weakly closed graphs has been introduced in [11] as a generalization of closed graphs. The final result of section 2 shows that each weakly closed graph is $m$-closed for some $m \leqslant 4$ (see Theorem 12). In Section 3 we study 3-closed property of trees and we show that a tree $T$ with $n$ vertices is 3 -closed if and only if it is not a path and there exists a labeling of its vertices such that $d(i, i+1) \leqslant 2$ for each $i<n$ (see Theorem 13). The class of 3-closed trees and the number of elements of the reduced Gröbner basis of $J_{T}$ for a 3-closed labeling is also studied by means of the bipartite graph $G^{*}$ attached to a simple graph $G$ corresponding to the generators of $J_{G}$ (see Definition 16, Theorem 17 and Corollary 18).

In Section 4, we study a class of trees constructed from caterpillar trees. We characterize the minimal primary decomposition of this class of trees (see Theorem 20). Also, we show that they are 3-closed. For some other trees constructed by caterpillar trees we show 3-closed property (see Theorem 21). To prove Theorem 21, we need an algorithm to give a 3-closed labeling to the vertices of a caterpillar tree such that 1 is assigned to an arbitrary vertex. This is provided in algorithm 22 presented in the Appendix section.

In the following, we review some definitions and results from [9] which we need in the next sections.

Definition 1. Let $G$ be a simple graph on $[n]$, and let $i$ and $j$ be two vertices of $G$ with $i<j$. A path $i=i_{0}, i_{1}, \ldots, i_{r}=j$ from $i$ to $j$ is called admissible, if

(i) $i_{k} \neq i_{\ell}$ for $k \neq \ell$;

(ii) for each $k=1, \ldots, r-1$ one has either $i_{k}<i$ or $i_{k}>j$;

(iii) for any proper subset $\left\{j_{1}, \ldots, j_{s}\right\}$ of $\left\{i_{1}, \ldots, i_{r-1}\right\}$, the sequence $\left\{i, j_{1}, \ldots, j_{s}, j\right\}$ is not a path.

Given an admissible path $\pi: i=i_{0}, i_{1}, \ldots, i_{r}=j$ from $i$ to $j$, where $i<j$, we associate the monomial

$$
u_{\pi}=\left(\prod_{i_{k}>j} x_{i_{k}}\right)\left(\prod_{i_{\ell}<i} y_{i_{\ell}}\right) .
$$

By [3, Chapter 2, Proposition 6], the reduced Gröbner basis of $J_{G}$ with respect to $\prec$ is unique. We have: 
Theorem 2. [9, Theorem 2.1] Let $G$ be a simple graph on $[n]$. Then the set of binomials

$$
\mathcal{G}=\bigcup_{i<j}\left\{u_{\pi} f_{i j}: \pi \text { is an admissible path from } i \text { to } j\right\}
$$

is the reduced Gröbner basis of $J_{G}$.

By [9, Theorem 3.2], we can write $J_{G}$ as a finite intersection of prime ideals. In fact, corresponding to each subset $S \subset[n]$ we have the prime ideal

$$
P_{S}(G)=\left(\bigcup_{i \in S}\left\{x_{i}, y_{i}\right\}\right)+J_{\tilde{G}_{1}}+\cdots+J_{\tilde{G}_{c(S)}},
$$

where $G_{1}, \ldots, G_{c(S)}$ are the connected components of the induced subgraph on the vertices $[n] \backslash S$, and $\tilde{G}_{\ell}$ is the complete graph on the vertices of $G_{\ell}$ for all $\ell$. Then

$$
J_{G}=\bigcap_{S \subset[n]} P_{S}(G) .
$$

Moreover, $\operatorname{dim} R / J_{G}=\max \{(n-|S|)+c(S): S \subset[n]\}$ and hence $\operatorname{dim} R / J_{G} \geqslant n+c(G)$, where $c(G)$ is the number of the connected components of $G$. Equation (1) also shows that $J_{G}$ is a radical ideal. If $G$ is a connected graph then $P_{\emptyset}(G)=J_{K_{n}}$ is a minimal prime ideal of $J_{G}$. Note that if $S$ is an arbitrary subset of $[n]$ the prime ideal $P_{S}(G)$ is not necessary a minimal prime ideal of $J_{G}$. The next lemma detects the minimal prime ideals of $J_{G}$ when $G$ is a connected graph. Note that for $S \subset[n]$, by $c(S)$ we mean $c\left(G_{[n] \backslash S}\right)$.

Lemma 3. [9, Corollary 3.9] Let $G$ be a connected graph on the vertex set $[n]$ and $S \subset[n]$. Then $P_{S}(G)$ is a minimal prime ideal of $J_{G}$ if and only if $S=\emptyset$, or $S \neq \emptyset$ and for each $i \in S$ one has $c(S \backslash\{i\})<c(S)$.

\section{$2 m$-closed graphs}

In this section we study the reduced Gröbner basis of $J_{G}$. As Theorem 2 shows the reduced Gröbner basis depends on the labeling of the vertices of $G$. We recall that a labeling of $G$ is a bijection $V(G) \simeq[n]=\{1, \ldots, n\}$, and given a labeling, we typically assume $V(G)=[n]$.

The graph $G$ is called closed with respect to the given labeling if $J_{G}$ has a quadratic Gröbner basis with respect to $\prec$. By $[9$, Theorem 1.1] we have:

Theorem 4. Let $G$ be a simple graph on the vertex set $[n] . G$ is closed if and only if the following condition is satisfied:

For every two edges $\{i, j\}$ and $\{k, \ell\}$ in $E(G)$ with $i<j$ and $k<\ell$, one has $\{j, \ell\} \in E(G)$ if $i=k$, and $\{i, k\} \in E(G)$ if $j=\ell$. 
Let $G$ be a graph, we recall that the clique complex of $G$, denoted $\Delta(G)$, is the simplicial complex on $[n]$ whose faces are the cliques of $G$. The graph $G$ is closed if and only if there exists a labeling of $G$ such that all facets of $\Delta(G)$ are intervals $[a, b] \subset[n]$ (see [6, Theorem 2.2]). Closed graphs are studied in more details in [2, 4].

Following the definition of closed graph we introduced $m$-closed graphs.

Definition 5. Let $m$ be a positive integer. We say that a graph $G$ with vertex set $V(G)=\left\{v_{1}, \ldots, v_{n}\right\}$ is $m$-closed, if its vertices can be labeled by $[n]$ such that for this labeling all the elements of $\mathcal{G}$ are of degree $\leqslant m$, and $m$ is the least integer with this property for $G$.

Moreover, a labeling of the vertices of $G$ is called an $m$-closed labeling if the reduced Gröbner basis of $J_{G}$ is in degree $m$ and less than $m$ with respect to this labeling.

By the above definition a closed graph is a 2-closed graph. the cycle $C_{4}$ (cycle with 4 vertices) is 3-closed and $C_{5}$ is 4 -closed.

By Theorem 2, a graph $G$ is $m$-closed if and only if, there exists a labeling for its vertices such that each admissible path in $G$ has at most $m$ vertices and in each labeling of the vertices, there exists an admissible path of length $\ell$ where $\ell \geqslant m-1$.

We recall that a bridge is an edge whose removal from a graph increases the number of components. If $e$ is a bridge of a connected graph $G$, and $H_{1}$ and $H_{2}$ are the connected components of $G \backslash e$, we write $G \backslash e=H_{1} \sqcup H_{2}$.

In the following we find some information about $m$-closed graphs.

Proposition 6. (i) Let $G$ be a graph and $\ell$ be the length of the longest induced path of $G$. Then $G$ is $m$-closed for some $m \leqslant \ell+1$.

(ii) Let $G$ be a graph and $H$ be an $\ell$-closed induced subgraph of $G$. Then $G$ is $m$-closed for some $m \geqslant \ell$.

(iii) Let $e$ be a bridge of a connected graph $G$ and $G \backslash e=H_{1} \sqcup H_{2}$. If $H_{1}$ is $m$-closed and $\mathrm{H}_{2}$ is $\ell$-closed $(\ell \geqslant m)$, then $G$ is $\ell$-closed provided that there exists an $m$-closed labeling of $H_{1}$ in which 1 is the label of the end point of $e$ in $H_{1}$ and there exists an $\ell$-closed labeling of $\mathrm{H}_{2}$ in which 1 is the label of the endpoint of e in $\mathrm{H}_{2}$.

Proof. Part (i) and (ii) follow from the definition of an admissible path and $m$-closed property.

For part (iii), assume that $H_{1}$ is an $m$-closed graph on $\left[n_{1}\right], H_{2}$ is an $\ell$-closed graph on $\left[n_{2}\right]$ and 1 is the label of the end points of $e$ in each $H_{i}(i=1,2)$. We give a labeling to $G$ by assigning to each vertex $i$ of $H_{1}$ the new label $n_{1}-i+1$ and to each vertex $i$ of $H_{2}$ the new label $n_{1}+i$. So, by this labeling $e=\left\{n_{1}, n_{1}+1\right\}$. It is easy to see that the graph $G=H_{1} \cup\left\{n_{1}, n_{1}+1\right\} \cup H_{2}$ is an $\ell$-closed graph on $\left[n_{1}+n_{2}\right]$.

A natural question to ask is that if the reduced Gröbner basis of $J_{G}$ has an element of degree $m$, can we conclude that it also has an element of degree $\ell$ for each $1<\ell<m$. This is not true in general, as the following example shows: 
Example 7. Let $G$ be the path on [5] with $E(G)=\{\{1,4\},\{3,4\},\{3,5\},\{2,5\}\}$. Then $\mathcal{G}$ has an element of degree 5 while it doesn't have any element of degree 4 .

For a simple graph $G$ on $[n]$, and $m \geqslant 3$, if the reduced Gröbner basis of $J_{G}$ has an element of degree $m$, then it has an element of degree 3. In fact, $G$ is not closed and by Theorem 4, there exist two edges $\{i, j\}$ and $\{i, \ell\}$ in $E(G)$ with $i<j, i<\ell$ and $\{j, \ell\} \notin E(G)$, or there exist two edges $\{i, j\}$ and $\{k, j\}$ in $E(G)$ with $i<j, k<j$ and $\{i, k\} \notin E(G)$. So $j, i, \ell$ or $i, j, k$ is an admissible path of length 2. So, $\mathcal{G}$ has an element of degree 3 .

Therefore, if $G$ is an $m$-closed graph, in each labeling of its vertices, there exists an admissible path of length 2. But as we have seen in the above example, we can not extend Theorem 4 to check if a labeling is a 3-closed labeling or not.

We recall that if $I$ is an ideal of $R$, the leading term ideal of $I$ with respect to $\prec$ is the monomial ideal of $R$ which is generated by $\left(L T_{\prec}(f) \mid 0 \neq f \in I\right)$ where $L T_{\prec}(f)$ is the leading term of $f$ with respect to $\prec$. We write $L T_{\prec}(I)$ for the leading term ideal of $I$.

If $G$ is a graph, it is clear that for any arbitrary labeling of the vertices of $G,|\mathcal{G}|=$ $\mu\left(L T_{\prec}\left(J_{G}\right)\right) \geqslant \mu\left(J_{G}\right)(\mu(I)$ is the minimal number of homogeneous generators of I). Moreover, $G$ is a closed graph if and only if there exists a labeling in which $\mu\left(\operatorname{LT}\left(J_{G}\right)\right)=$ $\mu\left(J_{G}\right)$. So If $G$ is a non-closed graph on $[n]$ and $\mu\left(L T_{\prec}\left(J_{G}\right)\right)=\mu\left(J_{G}\right)+1$, then $G$ is 3-closed.

It is well known by [9, Proposition 1.2], that a closed graph is chordal. In the following we find a generalization of this necessary condition for $m$-closed property. For this we need the following theorem about cycles:

Theorem 8. Let $C_{n}$ be the cycle on $n \geqslant 4$ vertices. Then $C_{n}$ is $m$-closed where $m= \begin{cases}\frac{n}{2}+1 & n \text { is even; } \\ \frac{n+1}{2}+1 & n \text { is odd. }\end{cases}$

Proof. Let $C_{n}$ be the cycle on $n$ vertices and $m$ be as defined in the theorem. To show the result, we first prove that in any labeling of the vertices of $C_{n}$, one can find an admissible path with at least $m$ vertices.

In an arbitrary labeling of the vertices of $C_{n}$, one of the following situation happens:

Case 1: For all $i \in\{1, \ldots, n-1\}, d(i, i+1)=1$. This case happens if and only if we give successive integers to the vertices. i. e., $\left(E\left(C_{n}\right)=\{\{1,2\},\{2,3\}, \ldots,\{n-\right.$ $1, n\},\{n, 1\}\})$. So $P: 1, n, n-1, \ldots, 3$ is an admissible path with $n-1$ vertices and $n-1 \geqslant m$.

Case 2: There exists $i \in\{1, \ldots, n-1\}, d(i, i+1)=\ell \geqslant 2$. So we have two admissible paths

$$
P_{1}: i, j_{1}, \ldots, j_{\ell-1}, i+1 \text { and } P_{2}: i, j_{1}^{\prime}, \ldots, j_{n-\ell-1}^{\prime}, i+1
$$

where $\{i, i+1\} \sqcup\left\{j_{1}, \ldots, j_{\ell-1}\right\} \sqcup\left\{j_{1}^{\prime}, \ldots, j_{n-\ell-1}^{\prime}\right\}=[n], P_{1}$ has $\ell+1$ vertices and $P_{2}$ has $n-\ell+1$ vertices.

In the case that $n$ is even, if $\ell+1<\frac{n}{2}+1$ and $n-\ell+1<\frac{n}{2}+1$, then $n+2<n+2$ which is a contradiction. So, one of the paths $P_{1}$ and $P_{2}$ has at least $m$ vertices. 
Now assume that $n$ is odd. Since $d(i, i+1)=\ell$, we have $\ell \leqslant n-\ell$. Moreover, $\ell=n-\ell$ if and only if $n=2 \ell$ which is a contradiction. So, $\ell<n-\ell$.

If $n-\ell+1<\frac{n+1}{2}+1$, then by $1+\ell<n-\ell+1<\frac{n+1}{2}+1$ we have $n+2<n+2$ which is a contradiction. So $P_{2}$ has at least $m$ vertices.

So in each labeling of the vertices of $C_{n}$, we have an admissible path with at least $m$ vertices.

Now, if we find a labeling of the vertices of $C_{n}$ such that each admissible path has at most $m$ vertices, the conclusion follows.

Suppose that:

$$
V\left(C_{n}\right)=\left\{v_{1}, v_{2}, \ldots, v_{n}\right\}, \quad E\left(C_{n}\right)=\left\{\left\{v_{1}, v_{2}\right\},\left\{v_{2}, v_{3}\right\}, \ldots,\left\{v_{n-1}, v_{n}\right\},\left\{v_{n}, v_{1}\right\}\right\}
$$

If $n$ is even, we do as follows:

1. $S=\left\{v_{1}, v_{2}, \ldots, v_{n}\right\}$,

2. label $v_{1}$ as 1 ,

3. $i=1$,

\section{While $i<n$ do}

(a) Pick $v_{j} \in S$ such that $d\left(i, v_{j}\right)=m-1$ and label $v_{j}$ as $i+1$,

(b) If $i+2<n$, label $v_{j+1}$ as $i+2$,

(c) $i:=i+2$.

By this labeling of the vertices, for each $i, d(i, i+1)=m-1$ if $i$ is odd and $d(i, i+1)=1$ if $i$ is even. So we have some admissible path with $m$ vertices.

If $n$ is odd, we do as follows:

For each $1 \leqslant i<m$, label $v_{i}$ as $2 i-1$ and for each $m \leqslant i \leqslant n$, label $v_{i}$ as $2(i-m+1)$. By this labeling, for each $i, d(i, i+1)=m-2$ and for each $i$ there is a unique admissible path with $m$ vertices between $i$ and $i+1$.

Now assume that $P: j_{1}, \ldots, j_{t}(t>m)$ is an admissible path in $C_{n}$. So, $j_{t}>j_{1}+1$.

If $n$ is odd, by the fact that $d(i, i+1)=m-2$ for each $i$, we conclude $j_{1}+1 \in V(P)$ which is a contradiction.

Assume that $n$ is even. If $j_{1}$ is odd, as above we conclude that $j_{1}+1 \in V(P)$ which is the desired contradiction. If $j_{1}$ is even and $j_{1}+1 \notin V(P)$, then $P^{\prime}=j_{1}+1, j_{1}, \ldots, j_{t-1}$ is a path with $t$ vertices. Since $d\left(j_{1}+1, j_{1}+2\right)=m-1, j_{1}+2 \in V\left(P^{\prime}\right)$. So $j_{t}>j_{1}+2$ and $j_{1}+2 \in V(P)$ again a contradiction.

The next corollaries are the generalization of the fact that a closed graph is chordal. These results are immediate consequences of Proposition 6 and Theorem 8.

Corollary 9. If $G$ is an $m$-closed graph, then each cycle of $G$ with $2 m-1$ or more vertices has a chord. 
Corollary 10. Let $G$ be an $m$-closed graph and

$$
\ell=\max \{t \mid \exists \text { an induced cycle with } t \text { vertices in } G\} \text {. }
$$

If $\ell \geqslant 4$, then $m \geqslant \begin{cases}\frac{\ell}{2}+1 & \ell \text { is even; } \\ \frac{\ell+1}{2}+1 & \ell \text { is odd. }\end{cases}$

A generalization of the notion of closed graph is weakly closed graph which has been introduced in [11]. Let $\mathrm{G}$ be a graph. $G$ is said to be weakly closed if there exists a labeling which satisfies the following condition: for all $i, j$ such that $\{i, j\} \in E(G), i$ is adjacentable with $j$ (for the definition of adjacentable see [11, Definition 1.2]. The following theorem is a characterization of weakly closed graphs.

Theorem 11. [11, Theorem 1.9] Let $G$ be a graph. Then the following conditions are equivalent:

1. $G$ is weakly closed.

2. There exists a labeling which satisfies the following condition: for all $i, j$ such that $\{i, j\} \in E(G)$ and $j>i+1$, the following assertion holds: for all $i<k<j$, $\{i, k\} \in E(G)$ or $\{k, j\} \in E(G)$.

In the following we relate the $m$-closed graphs to weakly closed graphs.

Theorem 12. Let $G$ be a weakly closed graph. Then $G$ is $m$-closed for some $m \leqslant 4$.

Proof. Suppose that $G$ is a weakly closed graph on $[n]$. Then by Theorem 11 , for all $i, j$ such that $\{i, j\} \in E(G)$ and $j>i+1$, the following assertion holds: for all $i<k<j$, $\{i, k\} \in E(G)$ or $\{k, j\} \in E(G)$.

We prove that each admissible path of $G$ has at most 4 vertices. Assume to the contrary that there exists an admissible path $P: i=i_{1}, i_{2}, \ldots, i_{m-1}, i_{m}=j$ with $m \geqslant 5$ vertices. Note that $i<j$. If $i_{2}>j$, then $i<j<i_{2}$ and $\left\{i, i_{2}\right\} \in E(G)$. So $\{i, j\} \in E(G)$ or $\left\{i_{2}, j\right\} \in E(G)$ which is a contradiction. If $i_{m-1}<i$, then $i_{m-1}<i<j$ and $\left\{i_{m-1}, j\right\} \in$ $E(G)$. So $\left\{i_{m-1}, i\right\} \in E(G)$ or $\{i, j\} \in E(G)$. Again, it is a contradiction. Therefore $i_{2}<j$ and $i_{m-1}>i$. Since $P$ is an admissible path, we have $i_{2}<i$ and $i_{m-1}>j$.

Let

$$
t=\min \left\{r \mid 2<r \leqslant m-1, i_{r}>j\right\} .
$$

So $i_{t-1}<i<j<i_{t}$ and $\left\{i_{t-1}, i_{t}\right\} \in E(G)$. If $t=3$, then $\left\{i_{2}, j\right\} \in E(G)$ or $\left\{j, i_{3}\right\} \in E(G)$ which is impossible because $m \geqslant 5$ and $P$ is an admissible path. If $t>3$, then $\left\{i_{t-1}, i\right\} \in$ $E(G)$ or $\left\{i, i_{t}\right\} \in E(G)$. This case also is impossible since $P$ is an admissible path.

So, in any case we get a contradiction. Thus $m \leqslant 4$ and the result follows.

Note that the converse of Theorem 12 is not true since $C_{5}$ is 4 -closed and not weakly closed. 


\section{3-closed trees}

In the following we characterize 3 -closed trees. Let $G$ be a simple graph on the vertex set $[n]$ and $\mathcal{G}$ has no element of degree more than 3 , then $d(i, i+1) \leqslant 2$ for each $i$. But the converse is not true in general. For example, let $C$ be the cycle on the vertex set $[n]$ and with the edge set $\{\{1,3\},\{3,4\},\{2,4\},\{2,5\},\{1,5\}\}$. Then for each $i, d(i, i+1) \leqslant 2$ but $C$ is 4 -closed.

We recall that by [9, Corollary 1.3], a tree is a closed graph if and only if it is a path. Next result shows that a 3 -closed labeling for a tree $T$ is a labeling in which $d(i, i+1) \leqslant 2$ for each $i$.

Theorem 13. Let $T$ be a tree with $n$ vertices and assume that $T$ is not a path. Then $T$ is 3-closed if and only if there exists a labeling for $V(T)$ such that $d(i, i+1) \leqslant 2$ for each $i$.

Proof. Assume to the contrary that there exists a tree $T$ on the vertex set $[n]$ such that $d(i, i+1) \leqslant 2$ for each $i$, and $T$ has an admissible path of length at least 3 . Let

$$
m-1=\max \{\ell(P) \mid P \text { is an admissible path }\}
$$

and

$$
i_{1}=\max \{t \mid \text { there exists an admissible path of length } m-1 \text { starting from } t\} .
$$

Then $m>3$ and we can consider an admissible path like $P: i_{1}, i_{2}, \ldots, i_{m}$. Since $T$ is a tree, $d\left(i_{1}, i_{m}\right) \geqslant 3$. So, $i_{1}+1 \neq i_{m}$ which shows that $i_{1}<i_{1}+1 \leqslant i_{m}-1<i_{m}$. Therefore $i_{1}+1 \notin\left\{i_{2}, i_{3}, \ldots, i_{m-1}\right\}$. Moreover, by $d\left(i_{1}, i_{1}+1\right) \leqslant 2$, one of the following situations happens:

Case a: $\left\{i_{1}, i_{1}+1\right\} \in E(T)$. In this case, $i_{1}+1, i_{1}, \ldots, i_{m}$ is an admissible path of length $m$ which is a contradiction by our choice of $m$.

Case b: $\left\{i_{1}+1, i_{2}\right\} \in E(T)$. In this case, $i_{1}+1, i_{2}, i_{3}, \ldots, i_{m}$ is an admissible path of length $m-1$ which is a contradiction by our choice of $i_{1}$.

Case c: There exists $j \in[n] \backslash\left\{i_{2}, \ldots, i_{m}\right\}$ such that $i_{1}+1, j, i_{1}$ is a path. In this case, consider the path $P^{\prime}: i_{1}+1, j, i_{1}, i_{2}, \ldots, i_{m}$. Since $\ell\left(P^{\prime}\right)=m+1$, by our choice of $m, P^{\prime}$ is not an admissible path. So, $i_{1}<i_{1}+1<j<i_{m}$. It is easy to see that $P^{\prime \prime}: j, i_{1}, i_{2}, \ldots, i_{m}$ is an admissible path of length $m$ which is again a contradiction by our choice of $m$.

Remark 14. By Theorem 13, a labeling of a tree $T$ is a 3-closed labeling if and only if $d(i, i+1) \leqslant 2$ for each $1 \leqslant i<n$. This is not true for an arbitrary 3-closed graph. For example, Let $G$ be a graph with

$$
V(G)=\left\{v_{1}, \ldots, v_{5}\right\} \quad \text { and } E(G)=\left\{\left\{v_{1}, v_{2}\right\},\left\{v_{2}, v_{4}\right\},\left\{v_{1}, v_{3}\right\},\left\{v_{3}, v_{4}\right\},\left\{v_{2}, v_{5}\right\}\right\} .
$$

Then $G$ is a bipartite 3 -closed graph. If we assign $i$ to each vertex $v_{i}$, then $d(i, i+1) \leqslant 2$ for each $1 \leqslant i<5$ but this is not a 3-closed labeling of $G$. 


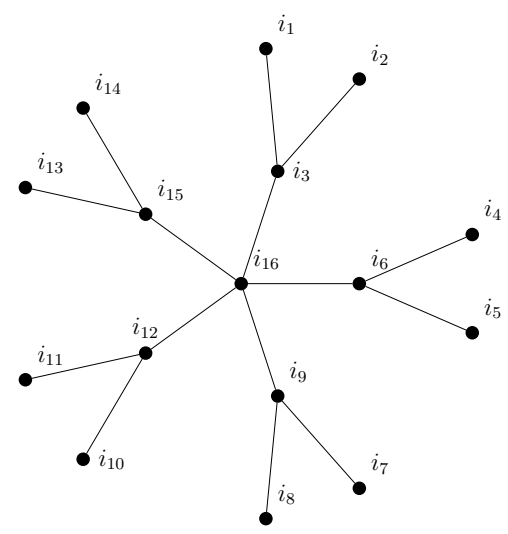

Figure 1:

Next we give an example of a tree which is not 3-closed.

Example 15. Consider the above tree on 16 vertices (Figure 1). We prove that $T$ is not 3-closed. By contradiction assume that there exists a labeling of $V(T)$ such that

$$
d(k, k+1) \leqslant 2 \text { for all } k \in\{1, \ldots, 15\} .
$$

Without loss of generality, we can assume that $\{1,16\} \cap\left\{i_{7}, i_{8}, \ldots, i_{15}\right\}=\emptyset$. So,

$$
\left\{i_{j}-1, i_{j}+1\right\} \subset\{1,2, \ldots, 16\} \text { for all } j \in\{7,8, \ldots, 15\} \text {. }
$$

If $i_{7}<i_{8}$ and they are not two successive integers, then by (2) $\left\{i_{7}-1, i_{7}+1, i_{8}+1\right\} \subseteq$ $\left\{i_{9}, i_{16}\right\}$ which is a contradiction. So, we can assume that $i_{8}=i_{7}+1$. By a similar argument, we should also have, $i_{11}=i_{10}+1$ and $i_{14}=i_{13}+1$.

Again, by (2) and (3) we can easily see that

$$
i_{16}=i_{7}-1 \text { or } i_{16}=i_{7}+2,
$$

and

$$
i_{16}=i_{10}-1 \text { or } i_{16}=i_{10}+2 \text {, }
$$

and

$$
i_{16}=i_{13}-1 \text { or } i_{16}=i_{13}+2 .
$$

So, $i_{7}=i_{10}$ or $i_{7}=i_{13}$ or $i_{10}=i_{13}$ which is a contradiction.

Definition 16. Let $G$ be a graph on the vertex set $[n]$, we associate to $G$ a bipartite graph $G^{*}$ where

$$
V\left(G^{*}\right)=\left\{x_{1}, \ldots, x_{n}\right\} \sqcup\left\{y_{1}, \ldots, y_{n}\right\}, \quad E\left(G^{*}\right)=\left\{x_{i} y_{j} \mid\{i, j\} \in E(G) \text { and } i<j\right\} .
$$

Note that if $G$ is a closed graph, for a closed labeling of $G, L T_{\prec}\left(J_{G}\right)=I\left(G^{*}\right)$ where $I\left(G^{*}\right)$ is the edge ideal of the graph $G^{*}$.

Conversely, if $H$ is a bipartite graph on the vertex set $\left\{x_{1}, \ldots, x_{n}\right\} \sqcup\left\{y_{1}, \ldots, y_{n}\right\}$ such that for each $\left\{x_{i}, y_{j}\right\} \in E(H)$ we have $i<j$, then we can associate to $H$ a simple graph $H_{*}$ on the vertex set $[n]$ in a natural way $\left(\left(H_{*}\right)^{*}=H\right)$. 
Note that if $T$ is a tree, then $T^{*}$ is also a tree. In the following, we give a characterization of 3-closed trees by means of Definition 16 .

Theorem 17. Let $\mathcal{T}_{n}$ be the set of all bipartite graphs $H$ on the vertex set $\left\{x_{1}, \ldots, x_{n}\right\} \sqcup$ $\left\{y_{1}, \ldots, y_{n}\right\}$ with the following properties:

1. $\left\{x_{i}, y_{j}\right\} \in E(H) \Longrightarrow i<j$.

2. for all $i \in\{1, \ldots, n-1\}$ one of the following conditions holds:

- $\left\{x_{i}, y_{i+1}\right\} \in E(H)$.

- There exists $j>i+1,\left\{x_{i}, y_{j}\right\},\left\{x_{i+1}, y_{j}\right\} \in E(H)$.

- There exists $j<i,\left\{x_{j}, y_{i}\right\},\left\{x_{j}, y_{i+1}\right\} \in E(H)$.

3. $|E(H)|=n-1$

Then a tree with $n$ vertices is 3-closed if and only if $T$ is not a path and there exists $H \in \mathcal{T}_{n}$ such that $T \cong H_{*}$.

Proof. If $T$ is a 3 -closed graph on $[n]$, then, by Theorem $13, d(i, i+1) \leqslant 2, \forall 1 \leqslant i<n$. So $T^{*}$ satisfies condition 2. Since $|E(T)|=\left|E\left(T^{*}\right)\right|=n-1$, the conclusion follows from the fact that $T=\left(T^{*}\right)_{*}$.

Conversely, if $H$ satisfies condition 1 then $H_{*}$ is defined and is a graph on $[n]$. By condition 2 , in $H_{*}, d(i, i+1) \leqslant 2$ for each $i$ and moreover $H_{*}$ is connected. Now since $\left|E\left(H_{*}\right)\right|=n-1=\left|V\left(H_{*}\right)\right|-1, H_{*}$ is a tree. So, by Theorem $13, H_{*}$ is a 3 -closed tree.

In the next corollary, we find the number of elements of the reduced Gröbner basis of a 3 -closed tree.

Corollary 18. Let $T$ be a tree on the vertex set $[n]$ and $d(i, i+1) \leqslant 2$ for all $i \in$ $\{1, \ldots, n-1\}$. Then $|\mathcal{G}|=n-1+\beta_{13}\left(I\left(T^{*}\right)\right)$.

Proof. Let $G$ be a simple graph on $[n]$ and $K_{3}(G)=$ the number of triangles of $G$. Then by $[15$, Theorem 2.2$], \beta_{13}\left(J_{G}\right)=2 K_{3}(G)$. So, for an arbitrary tree $T, \beta_{13}\left(J_{T}\right)=0$.

Now, if $d(i, i+1) \leqslant 2$, then by Theorem $13, L T_{\prec}\left(J_{T}\right)$ is generated in degrees 2 and 3. So, $\beta_{23}\left(L T_{\prec}\left(J_{T}\right)\right)=0$ and

$$
\beta_{13}\left(L T_{\prec}\left(J_{T}\right)\right)=\beta_{13}\left(\left\langle x_{i} y_{j} \mid i<j,\{i, j\} \in E(T)\right\rangle\right)=\beta_{13}\left(I\left(T^{*}\right)\right) .
$$

Moreover, by [14] the graded Betti numbers of $J_{T}$ is obtained from the graded Betti numbers of $L T_{\prec}\left(J_{T}\right)$ by a sequence of consecutive cancelations. So

$$
\beta_{03}\left(L T_{\prec}\left(J_{T}\right)\right)=\beta_{13}\left(L T_{\prec}\left(J_{T}\right)\right)=\beta_{13}\left(I\left(T^{*}\right)\right)
$$

and the conclusion follows.

We remark that if $G$ is an arbitrary 3-closed graph, for a 3-closed labeling, the same argument as the proof of Corollary 18 shows that $|\mathcal{G}|=|E(G)|+\beta_{13}\left(I\left(G^{*}\right)\right)-2 K_{3}(G)$. 


\section{Binomial Edge Ideals of Caterpillar Trees}

In this section, we study the binomial edge ideals of caterpillar trees and some trees constructed from this kind of trees. First we recall its definition.

Definition 19. A caterpillar tree is a tree $T$ with the property that it contains a path $P$ such that any vertex of $T$ is either a vertex of $P$ or it is adjacent to a vertex of $P$.

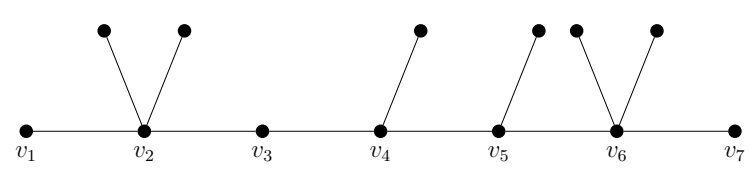

Figure 2:

Note that the path $P$ in the definition of a caterpillar tree is a longest induced path of $T$ and we call it the central path of $T$. Figure 2 is an example of a caterpillar tree with the central path $P: v_{1}, v_{2}, \ldots, v_{7}$.

Caterpillar trees were first studied by Harary and Schwenk [8]. These graphs have some applications in chemistry and physics [5].

Let $T$ be a caterpillar tree and $\ell$ be the length of its longest induced path. By $[6$, Theorem 1.1] $\operatorname{depth}\left(R / J_{T}\right)=|V(T)|+1$ and by [1, Theorem 4.1] $\operatorname{reg}\left(R / J_{T}\right)=\ell$. In the following we describe the minimal primary decomposition of $J_{T}$. We recall that since $J_{T}$ is a radical ideal, to know the minimal primary decomposition of $J_{T}$, it is enough to characterize its minimal prime ideals.

Theorem 20. Let $T$ be a caterpillar tree, $P: v_{1}, \ldots, v_{l}$ be the central path of $T$ and $S \subset V(T)$. Then $P_{S}(T)$ is a minimal prime ideal of $J_{T}$ if and only if $S=\emptyset$ or $S=$ $\left\{v_{i_{1}}, \ldots, v_{i_{k}}\right\} \subseteq\left\{v_{1}, \ldots, v_{l}\right\}$ where $1<i_{1}<\cdots<i_{k}<l$ satisfy the following conditions:

- If $\operatorname{deg}\left(v_{i_{j}}\right)=2$, then $d\left(v_{i_{j}}, v_{i_{j+1}}\right) \geqslant 2$ and $d\left(v_{i_{j}}, v_{i_{j-1}}\right) \geqslant 2$

- If $\operatorname{deg}\left(v_{i_{j}}\right)=3$, then $d\left(v_{i_{j}}, v_{i_{j+1}}\right) \geqslant 2$ or $d\left(v_{i_{j}}, v_{i_{j-1}}\right) \geqslant 2$.

Proof. We prove that each prime ideal corresponding to a set $S$, where $S$ is satisfying in the mentioned conditions, is a minimal prime ideal by induction on the number of vertices in the set $S$.

For $k=1$ the statement is obvious. Now assume theorem is true for each $S$ with $|S|=m$ and $S^{\prime}=\left\{v_{i_{1}}, \ldots, v_{i_{m+1}}\right\}$ has the mentioned conditions. If $S=\left\{v_{i_{1}}, \ldots, v_{i_{m}}\right\}$, by induction hypothesis, $P_{S}(T)$ is a minimal prime ideal of $J_{T}$. Let $d=\operatorname{deg}\left(v_{i_{m+1}}\right)$ and $d^{\prime}=\operatorname{deg}\left(v_{i_{m}}\right)$.

Depending on $d\left(v_{i_{m}}, v_{i_{m+1}}\right)$, we distinguish the following cases:

Case 1: $d\left(v_{i_{m}}, v_{i_{m+1}}\right) \geqslant 2$. In this case it is easy to see that $c\left(S^{\prime}\right)=c(S)+d-1$ and for all $j \in\{1, \cdots, m\}, c\left(S^{\prime} \backslash\left\{v_{i_{j}}\right\}\right)=c\left(S \backslash\left\{v_{i_{j}}\right\}\right)+d-1$. 
Case 2: $d\left(v_{i_{m}}, v_{i_{m+1}}\right)=1$. In this case, $d \geqslant 3$ and $d^{\prime} \geqslant 3$. A straightforward observation shows that $c\left(S^{\prime}\right)=c(S)+d-2$ and for all $j \in\{1, \ldots, m-1\}, \quad c\left(S^{\prime} \backslash\left\{v_{i_{j}}\right\}\right)=$ $c\left(S \backslash\left\{v_{i_{j}}\right\}\right)+d-2$. Moreover for deleting the vertex $v_{i_{m}}$, one of the following situations happens:

(a) $m=1$ or $d\left(v_{i_{m-1}}, v_{i_{m}}\right)=2$. One can see $c\left(S^{\prime} \backslash\left\{v_{i_{m}}\right\}\right)=c\left(S^{\prime}\right)-\left(d^{\prime}-2\right)$.

(b) $d^{\prime} \geqslant 4$. In this case, $c\left(S^{\prime}\right) \geqslant c\left(S^{\prime} \backslash\left\{v_{i_{m}}\right\}\right)+\left(d^{\prime}-3\right)$.

It is obvious that in all of the above situations, $c\left(S^{\prime} \backslash\left\{v_{i_{j}}\right\}\right)<c\left(S^{\prime}\right)$ for all $j \in$ $\{1, \cdots, m+1\}$. So, Lemma 3 implies $P_{S^{\prime}}(T)$ is a minimal prime ideal of $J_{T}$.

Now assume that $S \subset V(T)$ is not as described in the theorem. So, one of the following situation happens:

1) There exists a vertex $v$ of degree 1 in $S$. In this case, $c(S \backslash\{v\}) \geqslant c(S)$. So, by Lemma $3, P_{S}(T)$ is not a minimal prime ideal of $J_{T}$.

2) For some $j, \operatorname{deg}\left(v_{i_{j}}\right)=2$, and $\left(d\left(v_{i_{j}}, v_{i_{j+1}}\right)=1\right.$ or $\left.d\left(v_{i_{j}}, v_{i_{j-1}}\right)=1\right)$. Without loss of generality assume that $d\left(v_{i_{j-1}}, v_{i_{j}}\right)=1$. Since $v_{i_{j-1}}$ and $v_{i_{j}}$ are connected through just one edge, removing the vertex $v_{i_{j}}$ doesn't change the number of connected components of $T_{V(T) \backslash S}$, meaning that $c\left(S \backslash\left\{v_{i_{j}}\right\}\right)=c(S)$. Again, by Lemma 3, $P_{S}(T)$ is not a minimal prime ideal of $J_{T}$.

3) For some $j, \operatorname{deg}\left(v_{i_{j}}\right)=3, d\left(v_{i_{j}}, v_{i_{j+1}}\right)=1$ and $d\left(v_{i_{j}}, v_{i_{j-1}}\right)=1$. In this situation also straightforward observation shows that $c\left(S \backslash\left\{v_{i_{j}}\right\}\right)=c(S)$. So, $P_{S}(T)$ is not a minimal prime ideal of $J_{T}$.

So the conclusion follows.

For example, if $T$ is the caterpillar tree described in Figure 2, then by Theorem 20, it is easy to find all minimal prime ideals of $J_{T}$ and see that $\operatorname{dim}\left(R / J_{T}\right)=19$.

Finally, we prove that caterpillar trees and some trees constructed by caterpillar trees are 3-closed.

Theorem 21. (a) Let $T$ be a caterpillar tree. Then $T$ is 3-closed.

(b) Let $T=T_{1} \cup B \cup T_{2}$ where $T_{1}$ and $T_{2}$ are two caterpillar trees and $B$ is a bridge between $T_{1}$ and $T_{2}$, and the endpoints of $B$ are chosen from the vertices of the central paths of $T_{1}$ and $T_{2}$ respectively. Then $T$ is 3-closed.

More generally,

(c) Let $T$ be a tree and $T=T_{1} \cup B \cup T_{2}$ where $T_{1}, T_{2}$ and $B$ are caterpillar trees, and the endpoints of the central path of $B$ are chosen from the vertices of $T_{1}$ and $T_{2}$ respectively. Then $T$ is 3-closed.

Proof. (a) Let $n=|T|$, it is enough to find a labeling of $V(T)$ such that $d(i, i+1) \leqslant 2$ for each $1 \leqslant i<n$. 
Let $P: v_{1}, \ldots, v_{\ell}$ be the central path of $T$, and for each $1 \leqslant j \leqslant \ell, N_{T}^{\prime}\left(v_{j}\right)=$ $N_{T}\left(v_{j}\right) \backslash V(P)$ is determined the leaf neighbors of the vertex $v_{j}$.

We do as follows:

label $v_{1}$ as $1 ; t=2 ; j=2$;

While $j \leqslant \ell$ do

label $v_{j}$ as $t ; t=t+1$;

$S:=N_{T}^{\prime}\left(v_{j}\right)$;

While $S \neq \emptyset$ do;

$v:=$ pick $v \in S$ such that $v$ is the rightmost leaf of $v_{j}$;

label $v$ as $t$;

$t=t+1 ; S=S \backslash\{v\} ;$

end;

$j=j+1$

end

It is easy to see that by this labeling of $V(T), d(i, i+1) \leqslant 2$ for all $1 \leqslant i<n$.

(b) By proposition 6, it is enough to show that for each caterpillar tree $T$ and each vertex $v$ of its central path, there exists a 3-closed labeling in which 1 is assigned to $v$. This fact follows from Algorithm 22.

(c) Without loss of generality, we can assume that the endpoints of the central path of $B$ are chosen from the vertices of the central paths of $T_{1}$ and $T_{2}$ respectively. Because if this is not the case and for example $\{v\}=V\left(T_{1}\right) \cap V(B)$ where $v$ is not a vertex of the central path of $T_{1}$, then there exists a vertex $w$ of the central path of $T_{1}$ such that $e=\{v, w\} \in E\left(T_{1}\right)$. So we can replace $T_{1}$ with $T_{1} \backslash e$ and $B$ with $B \cup e$. We can also assume that $E\left(T_{1}\right), E\left(T_{2}\right)$ and $E(B)$ are pairwise disjoint sets.

Let $v \in V(B) \cap V\left(T_{1}\right)$ and $w \in V(B) \cap V\left(T_{2}\right)$. By Algorithm 22, there exists a 3-closed labeling of $V\left(T_{1}\right)$ that assigns $n_{1}=\left|V\left(T_{1}\right)\right|$ to $v$. By part (a) of the proof there exists a 3-closed labeling of $V(B)$ with integers $n_{1}, \ldots, n_{2}=n_{1}+|V(B)|-1$ that assigns $n_{1}$ to $v$ and $n_{2}$ to $w$. Again by Algorithm 22 there exists a 3-closed labeling of $V\left(T_{2}\right)$ with integers $n_{2}, \ldots, n_{3}=n_{2}+\left|V\left(T_{2}\right)\right|-1$ that assigns $n_{2}$ to $w$. All together we get a 3-closed labeling of $T$ and the conclusion follows.

By [11, Proposition 3.2], a tree $T$ is weakly closed if and only if $T$ is a caterpillar tree. So, by Theorem 21, If $T$ is a weakly closed graph, then $T$ is $m$-closed for some $m \leqslant 3$.

\section{Appendix}

In the following we introduce an algorithm to label the vertices of a caterpillar tree $T$ with integers $1, \ldots, n$ such that $d(i, i+1) \leqslant 2$ for all $1 \leqslant i<n$. Suppose that the central path of $T$ is $P: v_{1}, \ldots, v_{\ell}$ and for each $1 \leqslant j \leqslant \ell, N_{T}^{\prime}\left(v_{j}\right)=N_{T}\left(v_{j}\right) \backslash V(P)$ is determined the leaf neighbors of the vertex $v_{j}$. 
The algorithm works as follows. First a candidate for 1 is found by choosing an arbitrary vertex of the central path which is called $v_{i_{0}}$. We then go through the vertices in the central path. If $v_{i_{0}+1}$ has some leaf neighbors, we label them $2, \ldots, t$ from right to left, and then we label $v_{i_{0}+2}$ as $t+1$. Otherwise we label $v_{i_{0}+2}$ as 2 . Then we set $j=i_{0}+2$ and this process is repeated for the next vertices of the $v_{j}$ until we reach the endpoint of $P$. In the return path from $v_{l}$ to $v_{1}$ and then from $v_{1}$ to $v_{i_{0}}$ the similar process is repeated until every vertex is labeled.

Algorithm 22. Labeling algorithm of caterpillars trees:

Input: A caterpillar tree $T$ with the central path $P: v_{1}, \ldots, v_{\ell}$.

Output: A 3-closed labeling of $T$

$v_{i_{0}}:=$ one of the vertices on the central path;

$j:=i_{0} ;$ label $v_{i_{0}}$ as $1 ; t:=2$;

While $j<\ell-1$ do

$S:=N_{T}^{\prime}\left(v_{j+1}\right)$;

While $S \neq \emptyset$ do;

$v:=$ pick $v \in S$ such that $v$ is the rightmost leaf of $v_{j+1}$; label $v$ as $t$;

$t=t+1 ; S=S \backslash\{v\}$;

end;

label $v_{j+2}$ as $t$

$j=j+2 ; t=t+1$;

end

If $j==\ell-1$

label $v_{\ell}$ as $t$

$j=l ; t=t+1$;

\section{Otherwise}

label $v_{\ell-1}$ as $t$;

$j=\ell-1 ; t=t+1$

end

While $j>2$ do

$S:=N_{T}^{\prime}\left(v_{j-1}\right)$;

While $S \neq \emptyset$ do;

$v:=$ pick $v \in S$ such that $v$ is the rightmost leaf of $v_{j-1}$; label $v$ as $t$;

$t=t+1 ; S=S \backslash\{v\} ;$

end;

label $v_{j-2}$ as $t$;

$j=j-2 ; t=t+1$;

end

If $j==2$ and $i_{0}>1$

label $v_{1}$ as $t$;

$j=1 ; t=t+1$;

\section{Otherwise}

If $i_{0}>2$

label $v_{2}$ as $t$; 
$j=2 ; t=t+1 ;$

end;

end ;

While $j<i_{0}-2$ do

$S:=N_{T}^{\prime}\left(v_{j+1}\right)$;

While $S \neq \emptyset$ do;

$v:=$ pick $v \in S$ such that $v$ is the rightmost leaf of $v_{j+1}$; label $v$ as $t$;

$t=t+1 ; S=S \backslash\{v\} ;$

end;

label $v_{j+2}$ as $t$;

$j=j+2 ; t=t+1$;

end

If $j==i_{0}-2$

$S:=N_{T}^{\prime}\left(v_{i_{0}-1}\right)$;

While $S \neq \emptyset$ do

$v:=$ pick $v \in S$ such that $v$ is the rightmost leaf of $v_{i_{0}-1}$; label $v$ as $t$;

$t=t+1 ; S=S \backslash\{v\}$

end;

end;

Remark 23. If one wants to give a 3 -closed labeling to a caterpillar tree $T$ in such a way that 1 is assigned to $v \in N_{T}^{\prime}\left(v_{i_{0}}\right)$ for some $1<i_{0}<\ell$, it is enough to label $v$ as $1, v_{i_{0}}$ as 2 , set $N_{T}^{\prime}\left(v_{i_{0}}\right)=N_{T}^{\prime}\left(v_{i_{0}}\right) \backslash\{v\}$ and start with $t:=3$ instead of $t:=2$.

Moreover, if one wants to give a 3-closed labeling to a caterpillar tree $T$ in such a way that $n=|V(T)|$ is assigned to an arbitrary vertex $v$, it is enough to apply Algorithm 22, by labeling $v$ as 1 and at the end changing the label $i$ of each vertex to $n-i+1$.

Example 24. Here, we give an example of a labeled caterpillar tree using Algorithm 22. Note that 12 is the label of $v_{1}, 11$ is the label of $v_{2}, 1$ is the label of $v_{3}$ and so on .

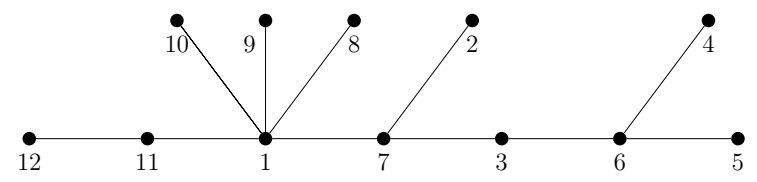

Figure 3:

Finally, we give an example of a 3-closed tree described in Theorem 21(part b). Note that the labeling is given by Algorithm 22, and Proposition 6 . 


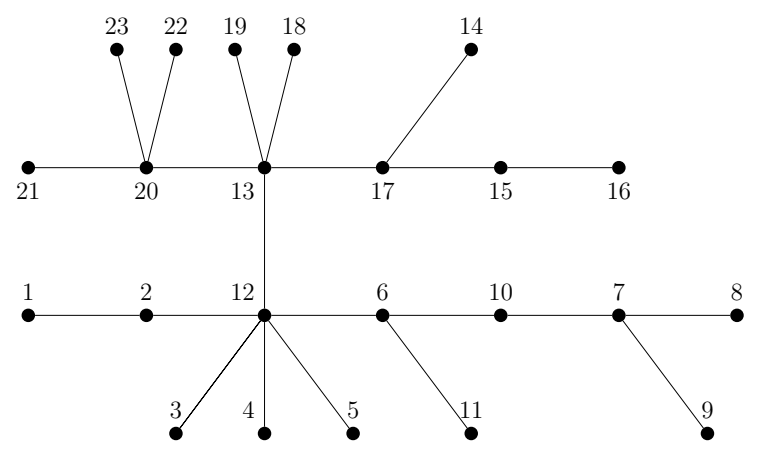

Figure 4:

\section{Acknowledgements}

We are very grateful to the anonymous referee for suggesting us Theorem 12 .

\section{References}

[1] F. Chaudhry, A. Dokuyucu, R. Irfan. On the binomial edge ideals of block graphs. arXiv:1402.2041v1.

[2] D. A. Cox, A. Erskine. On closed graphs. arXiv:1306.5149.

[3] D. Cox, J. Little, D, O'Shea. Ideals. Varieties, and Algorithms: An Introduction to Computational Algebraic Geometry and Commutative Algebra. Third Edition, Springer (2007).

[4] M. Crupi, G. Rinaldo. Binomial edge ideals with quadratic Gröbner bases. Electronic J. Combinatorics, 18(1):\#P211, 2011.

[5] S. EI-Basil. Applications of caterpillar trees in chemistry and physics. J. Math. Chem, 1(2):153-174, 1987.

[6] V. Ene, J. Herzog, T. Hibi. Cohen-Macaulay binomial edge ideals. Nagoya Math. J., 204:57-68, 2011.

[7] V. Ene, A. Zarojanu. On the regularity of binomial edge ideals. Math. Nachr., 2014. doi:10.1002/mana.201300186.

[8] F. Harary, A. J. Schwenk. The number of caterpillars. Discrete Math., 6(4):359-365, 1973.

[9] J. Herzog, T. Hibi, F. Hreinsdotir, T. Kahle, J. Rauh. Binomial edge ideals and conditional independence statements. Adv. Appl. Math, 45:317-333, 2010.

[10] D. Kiani , S. Saeedi Madani. The regularity of binomial edge ideals of graph. arXiv:1310.6126v2.

[11] K. Matsuda. Weakly closed graphs and F-purity of binomial edge ideals. arXiv: $1209.4300 v 2$. 
[12] K. Matsuda, S. Murai. Regularity bounds for binomial edge ideals. J. Commut. Algebra, 5(1):141-149, 2013.

[13] M. Ohtani. Graphs and Ideals generated by some 2-minors. Comm. in Algebra 39:905-917, 2011.

[14] I. Peeva. Consecutive cancellations in Betti numbers. Proc. Amer. Math. Soc., 132(12):3503-3507, 2004.

[15] S. Saeedi Madani, D. Kiani. Binomial edge ideals of graphs. Electronic J. Combinatorics 19(2):\#P44, 2012. 